\title{
Real-time Video Enhancement and Computer Vision for In-Vivo Microscopy
}

Hany Osman ${ }^{1}$

1. Indiana University, Department of Pathology and Laboratory Medicine, Indianapolis, Indiana, USA

In-vivo microscopy techniques are increasingly used for clinical applications and diagnostics. Among the most common utilized technologies are microscopes based on imaging fiber optic bundles. Fiber optic bundles are used to transpose images from the tissue, through an endoscope, to the objective lens of a microscope. These fiber bundles are composed of thousands of smaller optical fibers bundled together to form a probe where each fiber acts as a pixel. One of the main drawbacks of this technique is the resulting honeycomb artefact superimposed on images transmitted through the bundle [1]. In addition to the honeycomb artefact, images also lose some contrast and/or brightness. Multiple algorithms have been proposed to enhance in-vivo images [1]. Unfortunately, most of the algorithms provide enhancement after the acquisition of the images or videos has occurred, which in some sense, defeats the purpose in-vivo imaging in some instances.

In the current paper we propose the ability of customized software in enhancing video feeds in real-time allowing for enhanced interpretability at the point of acquisition. We developed software using the software development platform; Liveblox, to process enhanced video feed using an algorithmic approach.

To demonstrate the applicability of this technique, we applied Gaussian spatial smoothing followed by basic equalization to practically eliminate the honeycomb effect in real-time and enhance the image (Fig.1, Fig.2). Additional computer vision functions including pixel counts, stabilization and motion estimation can potentially offer real-time analytical functions such as estimation of nuclear size or cellularit. These functions can dramatically increase the ability of providing accurate diagnoses. Additionally pseudo-coloring of the videos and images can be achieved potentially providing real-time video feed similar to those of standard histology with hematoxylin and eosin staining.

References:

[1] Han JH et al, Optics express 18 (7) (2010) 


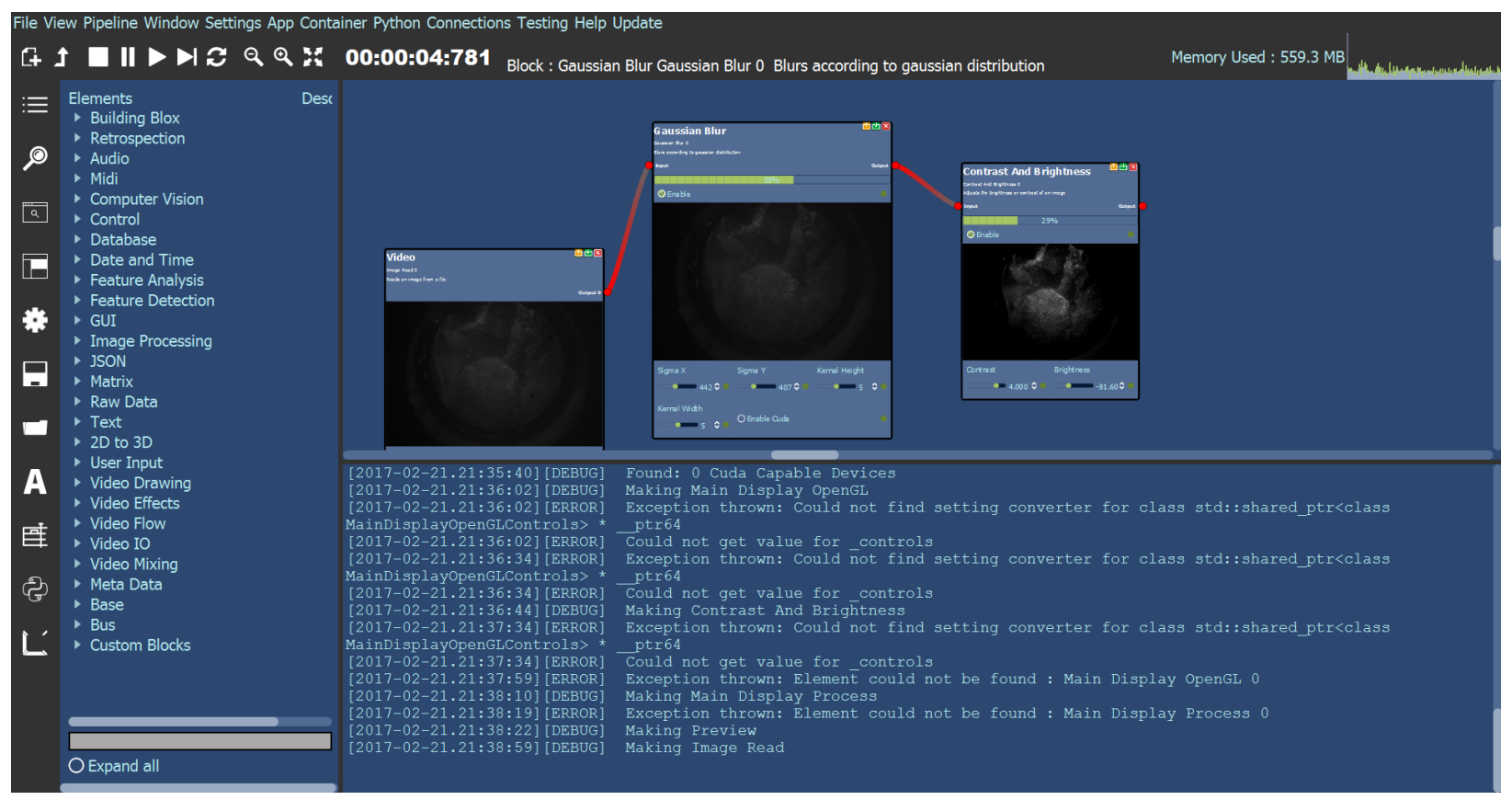

Figure 1. Simple algorithm in Liveblox showing acquisition, Guassian blur and image enhancement in real-time. Resulting videos are projected in full screen.

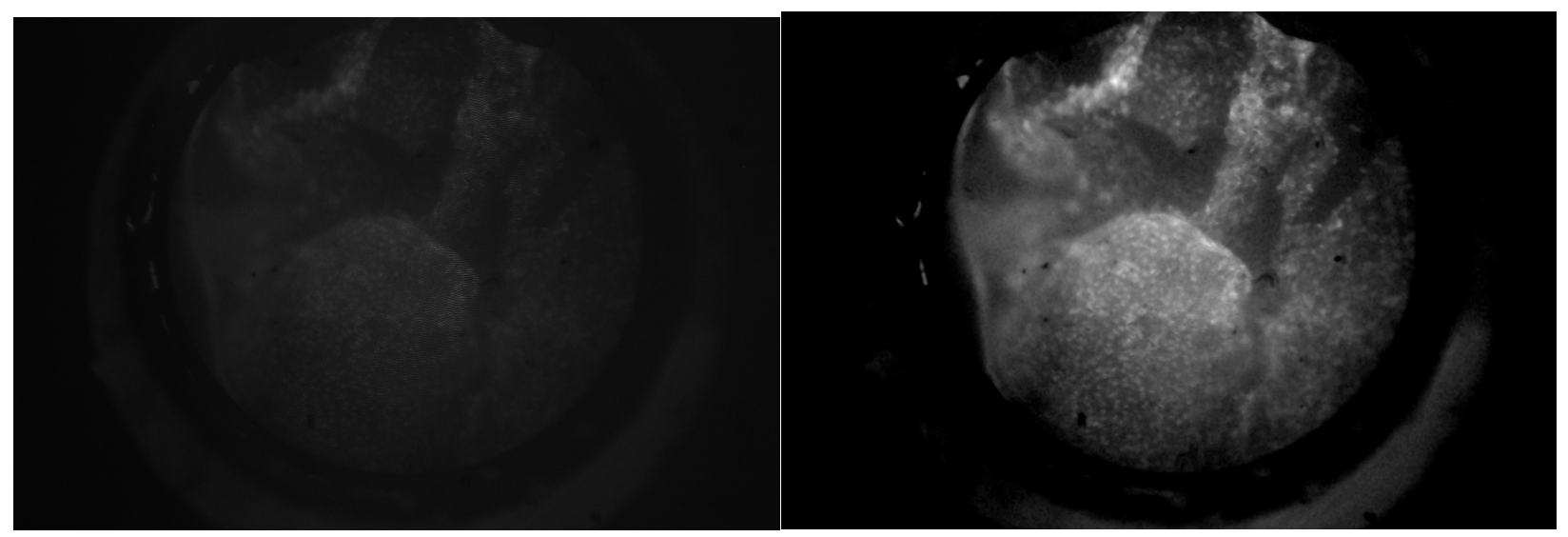

Figure 2. Ex-vivo video of a meningioma without enhancing exhibiting low luminance and artefacts (left). Real time enhanced video with honeycomb elimination and improved contrast (right) 